\title{
11. THE APPLICATION OF THE INTERNET AND INTRANETS IN BUSINESS
}

\author{
M.A.Murray, S. Kerridge, A. Slade, \\ D. Grey, I. Fergusson, J.B. Thompson.
}

\begin{abstract}
The Internet and its corporately owned counterpart, intranets, continue to prove fertile in spawning a wide variety of business applications. The superimposability of Internet/ intranet based solutions onto the needs of business is explored in this review, and asserted as a pivotal reason for this phenomenon. In terms of business utility the splicing of IT/IS and telecommunications that comprise networks has proven ever more useful in recent years making this technology strategically significant and demanding strategic management. The significance of generic categories of application is addressed with examples provided for illustration. These categories include communication, publishing and enterprise support. Problems of security are discussed and ways of circumventing these problems considered. Finally a summary is given in which is the importance of these networks to business is reasserted.
\end{abstract}

\section{Keywords:}

Electronic Commerce, Electronic Procurement, Internet, Intranet.

The original version of this chapter was revised: The copyright line was incorrect. This has been corrected. The Erratum to this chapter is available at DOI: 10.1007/978-0-387-35393-7_22 


\section{Introduction}

The history of the Internet from its inception as a US military project to its ubiquitous presence in the 1990's is well documented (Comer, 1994), yet given its proliferation in terms of the numbers of providers and users involved its scale is difficult to quantify accurately. The spread of corporately owned intranets has been every bit as prolific. Intranets have led to a re-evaluation of organisational processes to see where they can be used to cut costs and advance competitive advantage.

The open TCP/IP (Transmission Control Protocol / Internet Protocol) suite has allowed wide proliferation of the Internet/intranets. Diverse computer resources often cause discontinuity, in the form, for example, of users having to switch between systems to achieve their aims. The traversability promoted by unifying TCP/IP protocols is essential not only to the Internet but also large corporate intranets which often consist of diverse computer systems. Open protocols deployed on intranets can prove a more cost-effective method of intra-organisational networking than proprietary systems, (the alternative) which often lead to organisations being 'locked' to one vendor and limit that organisation's options to scale-up. Related to this, the formation of extranets (essentially an intranet connected to an external body) is more cost-effective via an Internet link rather than a point-to-point connection hired by the organisation. Cost-reduction is emphasised by Segal (Segal, 1996) as being instrumental in the Internet's adoption in recent years by so many users.

To begin with section 2 will explore the Internet/intranet paradigm. This will be considered in terms of its proliferation, it paradigmatic fit and its strategic fit in subsections 2.1,2.2 and 2.3 respectively. The business applications will be considered along with the security problems involved.

\section{Exploration Into The Internet/Intranets}

\subsection{Proliferation}

Figure 1 shows how Internet use has increased in recent years. The Internet has moved from being a specialist domain to being commonplace in a much wider arena.

A reflection of its popularity in Britain is the UK Prime Minister's (Tony Blair) promise to have every school in Britain connected as soon as possible, which is to be achieved by a partnership between government and telecommunications companies. To accompany this rise in popularity, there has been a rapid increase in the number of companies with intranet installations. Figure 2 shows that the sales of servers for intranets have over taken that for the Internet, which is consistent with Netscape's claim that $\mathbf{8 0 \%}$ of their revenue now comes from servers bound for corporate intranets. Shamoon (Shamoon, 1996) predicts that intranet popularity will increase further once the model assimilates all the functionality of groupware. 
Figure 1 Growth of Internet (Stobart,1995)
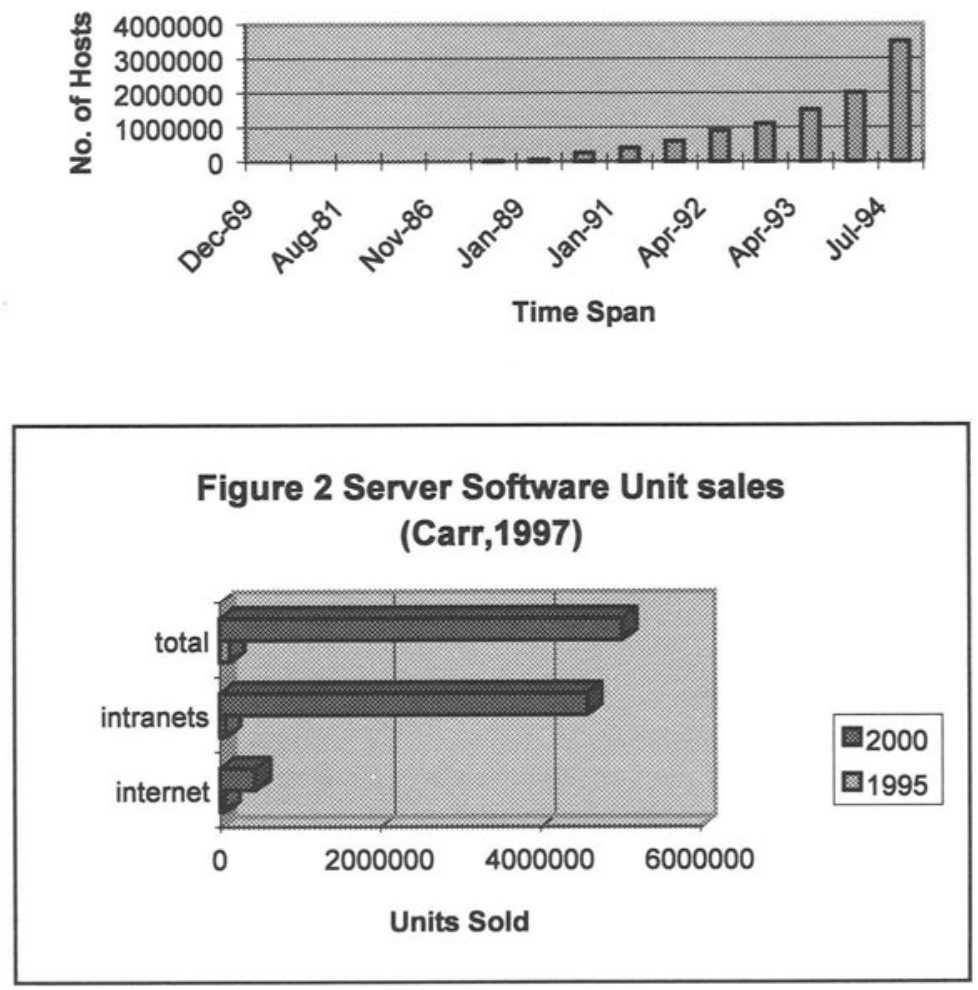

\subsection{Paradigmatic Fit}

This section looks at the Internet/intranet model as a useful tool in commerce.

The fusion of connectivity and ease of use that typifies today's Internet and intranets represents the further improvement of networking. It has emerged that intranets, in particular, provide versatility that can be applied effectively in many situations; Ward (1996) asserts that intranets represent the first time technology has mapped directly onto business needs both in terms of utility and cost. A small intranet can be established on an existing network simply by installing:-

- $\quad$ A TCP/IP router.

- A web server and browsers.

- An HTML editor

In IT/IS terms none of these items is particularly expensive. The software elements can often be acquired free of charge from the large vendors. It can be accepted a 
priority that this will be highly attractive to IT managers looking for solutions within the constraints of tight budgets.

Once an intranet is established, open standards ensure that it can be easily expanded throughout an organisation and if necessary can be used to connect geographically separated sites; basically the versatility and scalability of this model ensures that a suitable topology can be found . It is asserted that paradigmatic 'fit' of these networks or the superimposability of related solutions and business architecture advanced by the malleability of the paradigm, is the chief factor in their popularity. Business architecture (Hogbin \& Thomas, 1994) comprising data models and processes of an organisation, can be as dynamic and as multifarious as the organisations themselves. Adaptability of any system is of the essence, so clearly a system that uses open standards has advantage over proprietary systems, particularly once that system has gained wide acceptance the way TCP/IP protocols have. Further, TCP/IP support in many operating systems allows users to work in their preferred (native) environments, negating the need for extensive retraining.

\subsection{Strategic Fit}

By virtue of the wide scope of business applications they enable, consideration of these networks is elevated to the strategic level. The strategic significance of IT/IS, long recognised by writers (McFarlan,1984; Porter, 1985) requires that IT/IS be strategically managed if business is to fully exploit it for competitive advantage. Without getting into the minutia of the various models of IT strategy, many involve identifying development trajectories that can be used to make useful predictions regarding IT investment (Robson, 1997). Generally the strategic management of IT/IS is complicated by the mutable nature of the underlying technology which makes strategic management difficult. In such an environment traditional accounting methods for appraising investments such a ROI or NPV are not always suitable for IT investments because of the difficulties in quantifying the benefits of IT (Walsham, 1993).

\section{Applying The Technology In Business}

Much of the utility of intranets comes from their ability to disseminate information rapidly throughout an organisation. Organisations have been involved in the acquisition and the distribution of information for centuries. This dissemination, of information (the lifeblood of most organisations) has been speeded up by the technologies of the Information Society and is now being catalysed by Internet / intranets. The first organisations to utilise the technology did so in mission-critical areas; The American video company Home Box Office (HBO) used an intranet to distribute its promotional materials to its many salespeople, effecting an improvement on distributing the same materials as hard copy. There are numerous other examples in the literature (Shepherdson, 1997) of how this technology is being deployed in mission-critical areas. 
Three areas of application will be considered here:-

- Publishing Documentation

- Communication

- Enterprise Support

\subsection{Publishing Documentation.}

Often an organisation's first venture into this technology will be a publishing project -often just a telephone directory. Affordable storage media such as large hard disks and writable CD ROMs ensure that even small intranets make perfectly adequate repositories of all types of content. The London Underground Corporation in the UK recently published a huge 13 volume safety manual on an intranet as part of a pilot scheme to assess the viability of a corporate intranet. This scheme relied on the willingness of the of users to pull information from the intranet as opposed to having it 'pushed' as hard copy. The scheme was reported a success (Ward, 1996). Ultimately the bulk of an organisation's documentation can be moved onto an intranet and a 'workflow' system be introduced (Vasali, 1997). Workflow systems are an attempt at computerised storage, manipulation and management of documentation that facilitate monitoring of products and resources as well aiding accountancy tasks such as invoicing and undertaking audit trails. The efficacy of documentation publishing on the Internet / intranets is furthered by HTML links and search engines that allow complex searches to be made to enable the locating of specific information. Naturally some materials are more suitable to network publishing than others; likely candidates include, materials that can completely substitute the printed page, materials that are frequently updated and materials such as reference works that can take good advantage of hypertext links

(PTWEB, 1996).

Though the publication of materials on the Internet is limited by confidentiality of much corporate material, there still is good scope for certain types of publication.

The infinite storage capacities of the Internet make it perfect for storing huge catalogues that can be used in Electronic Commerce, allowing consumers to assess products online. Problems regarding on-line payments have not been completely resolved, yet governments across the globe, including those in the EU and the USA, are sponsoring initiatives to encourage the use of e-commerce - recognising its significance in modern economies. The attraction to producers of having their products displayed in virtual outlets on the Internet is immediately apparent when the number of potential browsers is considered. Anderson (1997) informs us that there is a great deal more business to business e-commerce than consumer ecommerce, with electronic procurement and tendering becoming widespread.

As well as representing a new departure in terms of speed and distribution capability,electronic publishing is important because of the amount of materials it saves. The potential for saving paper copy is massive. Take, for instance the case of a large organisation issuing an Invitation to Tender. Rather than send huge volumes 
out to each interested party the company can publish the Invitation on the Internet and tender can be made electronically.

Advanced transmission methods such as UDP and TCP multicasting (Dyson et al 1997) and improvements in modems and ISDN have ensured that publishing is not restricted to static content. Animation, audio and video can now be added to static content and accessed by any user with a browser with the required capability.

\subsection{Communication tools}

Communication at all levels is enhanced by Internet/intranets. Inter departmental communication is enhanced by the intranets while communication between subsidiaries is enhanced by linking their respective intranets. In this context these networks represent a continuation of the marriage between telecommunications and IT that has led to such a radical increase in the availability of information (Forrester, 1985) over the last few decades.

The most obvious way in which these networks aid communication is via e-mail and newsgroups, both of which benefit from the robustness of the TCP/IP suite. Newsgroups help people who would not normally meet to communicate and discuss/exchange ideas, while e-mail permits instant communication with anyone connected to the Internet with an e-mail account. Each of these tools is now taken for granted yet their importance as an enabler of communication should not be understated.

Increasing bandwidth also permits the use of video conferencing which uses various Electronic Meeting Systems (EMS) to allow real-time conferencing across networks. An extensive survey by Pollard (1996) revealed that many large organisations including IBM, American Express and the Royal Bank of Canada are now deploying EMS and that moreover it is becoming more extensively used. The survey found that managers saw advantages in EMS including efficient use of time, the promotion of organisational flexibility, and the creation of greater scope for collaborative working. Given improvements in transmission and the wider acceptance by the business community of Internet technology, there is no reason why this technology should not become more prevalent.

To deny the significance of the Internet/intranets to communication is difficult even after discounting the enthusiasm reflected in companies rushing to install intranets and in literature replete with panegyrics and superlatives. The use of the Internet has led to the term 'Global Village' being used to describe a world community of users constantly in communication with each other. Intranets allow the formation of corporate villages in which communication in the organisation is enhanced. Further many management writers (Burnes, 1997; Mullins, 1996) expound the importance of communication in organisations especially at times of change.

\subsection{Enterprise Support}

Enterprise support is (of necessity) a broad category encompassing many forms of organisational behaviour. This section could be broken down into subsections on phenomena such as electronic commerce, electronic procurement and banking, but 
given the taxonomical uncertainties inherent in these nascent fields, and the scope of this paper, the broader category will suffice.

Any process involving transmission of information can benefit from networking. Existing applications can benefit by being integrated with the intuitive browser interfaces and by having their use extended across operating platforms. Additionally the diversity of Internet/intranet compatible applications is increasing; intranet compatible databases alone include Oracle, Sybase and DB2, while ODBC (Open Database Connectivity) conformance ensures that many other systems can share data across networks. Products such as JAVA, ActiveX and Livewire have been produced by the big players viz., Sun, Microsoft and Netscape respectively to provide a broader range of application programs that will function across these networks.

As well as a diversity of applications produced for this paradigm users are beginning to find new ways of applying the paradigm itself to enhance their business processes. James (1996) provides a good example of this. James relates the case of the American telecommunications giant AT\&T who used an intranet to reduce the number of their customer support line from 23 to 1 . Having 23 lines was seen as wasteful of both employee time and customer time, with customers often having to be passed from one person to another until their problem was solved. An intranet was introduced to allow telephonists to share information pertaining to many different problem areas. The intranet used a standard web browser for finding information and this required little retraining of employees. The scheme's success was down to the user's willingness to pull information from the intranet and share it with other users.

A second example, this time of the Internet being utilised concerns the field of corporate intelligence (Vine, 1997). Now that many organisations regularly publish materials on the Internet to achieve a corporate presence, there is now a great deal of information available for the perusal of interested competitors. The Internet provides the perfect platform for this type of intelligence activity, with millions of users constantly depositing and retrieving information it is debatably the biggest database in the world, with search engines boasting in-built Artificial Intelligence information on the most obscure subjects in readily accessed. Such practices naturally raise questions of confidentiality and morality.

James (1996) argues that intranets as well as being useful in functional areas of business but that they have the potential to revolutionise business organisation. $\mathrm{He}$ cites the use of intranets in the process of Business Reengineering, a process involving the reorganising of business with a view to improving efficiency. Many reengineering programs failed because of the lack of organisation-wide cohesion. The introduction of organisational intranets can counter this by improving communications and processing the huge volumes of data generated in this process. Of course, as well as re-engineering intranets can be usefully deployed in other management strategies e.g. TQM 


\section{Potential Security Problems Engendered By The Internet/Intranets}

When confidential materials are deposited on networks organisations must accept as corporate responsibility their security. There is a fine balance between allowing legitimate users access to conduct their business freely while at the same time stopping intruders. Internal threats can be inadvertent as well as malicious and include:-

- hardware threats such as theft or tampering

- Accidental deletion and theft of software, bugs and by viruses

- threats to sensitive data

Any organisation that is reliant on IS/IT, particularly in the form of networks which involve a good deal of shared data, must see to it that back-ups are routine and that disaster recovery is planned and regularly rehearsed. A system known as RAID (Redundant Array of Inexpensive Disks), whereby several small hard disks are used to store data as opposed to one large one, has been used to minimise the impact of disk failure.

Many systems have emerged to protect intranets with links to external networks, i.e. extranets, from external threat. 'Firewalls' are configurations of software and hardware that act as selective barriers monitoring use and denying access when necessary. For business on the Internet the primary method used in encryption.

It has to be emphasised that an intranet without a link to an external network is no more of a security risk than any type of corporate network. The choice of whether or not to conduct business on an intranet or an extranet (depending on how they're configured) will always be more secure; indeed the difference in scale between intranets and the Internet renders intranet security problems much more tractable.

Apropos of the Internet, its very public nature and size are cause for security concern. Several issues cause difficulties:-

- lack of ownership

- legislation cannot keep up with technological change

- human nature being what it is security will always be a problem on a public network.

What all this boils down to, is that security should be one of the first considerations of any company doing business on the Internet. 


\section{Summary}

The Internet and intranets are widely used by business for a wide variety of applications including processes that involve communication, publishing and enterprise support. Examples from Sunderland University are the Elpro and Supplypoint systems.

Elpro (Electronic Procurement for Europe) handles electronically the whole public procurement lifecycle. European procurement legislation requires public authorities to publish tenders above a certain value so that companies in all member states can bid for the work. Elpro handles the process from the initial Invitation to Tender, and PIN notices, through to contract award. Access is provided through the system to published public procurement information such as is currently available in such formats as Tenders Electronic Daily.

The Supplypoint project addresses the need of SMEs to match skills bases and form virtual companies to big for large contracts. SMEs participating in the system require the inclusion of a range of administrative services in the available skills base. These services include project management of large construction programmes, and also accounting and legal services dealing with the formation of virtual companies and the submission of bids. Supplypoint acts as a forum for SMEs to match skills, and locate partners with whom to bid for large contracts, and also incorporate the required range of administrative services into a virtual company. Supplypoint will link to the Elpro system, so that virtual companies can submit bids directly through the European procurement process.

The reason for the applicability of these networks is largely versatility which allows a huge rage of topologies and scale-up options. Behind this versatility lies the robust TCP/IP suite. As these technologies are widely adopted related security issues have to be addressed and resolved. Security threats have been shown to be both internal and external and can be inadvertent and malicious. Given the scope of security concerns, any organisation employing these networks must make sure security is reviewed and an appropriate security policy instigated.

\section{References}

Anderson, C. (1997) In Search of the Perfect Market, The Economist 14/9/97

Comer, S (1994) Internetworking with TCP/IP: Prentice Hall

Dyson, P, Coleman, P and Gilbert, L (1997) Intranets: Plan and Build an Effective Inranet, Alameda: Sybex.

Forester T. (1989) The Information Technology Revolution. Oxford: Basil Blackwell

Hogbin, G. and Thomas, D.V. (1994) Investing in Information Technology, Maindenhead: McGraw-Hill

James, G. (1996) Intranets Rescue Reengineering

$\mathrm{httm}: / / w w w . d a t a m a t i o n . c o m / P l u g i n /$ issues/1996/dec/12bpr.html

Accessed 20 February 1997

McFarlan, F.W. (1984) "Information Technology Changes The Way You Compete", Harvard

Business Review, May - June, pp. 98-103 
Netscape (1997) Intranets Redefine Corporate Information Systems. http://home.netscape.com/comprod/at_work/white_paper/indepth.html Accessed 15/07/97

PBTWEB (1996) Intranet Criteria. http://www.pbtweb.com/pbtweb/intranet.htm Accessed 15/07/97

Pollard, C.E. (1996) Electronic Meeting Systems: Journal of Systems Management May/June pp 22-28

Porter, M.E. (1985) "How Information Gives You Competitive Advantage", Harvard Business Review, July - August, pp. 149-160.

Robson, W. (1997) Strategic Management and Information Systems (2nd Ed.) London: Pitman Publishing

Segal,B(1996) A Short History of Internet Protocols at CERN http://wwwcn.cern.ch/pdp/ns/ben/TCPHIST.html Accessed 10 December 1997

Shamoon, D.N. Intranets Boost IS Buudgets, Datamation, Nov, 1996, pp 68-71.

Shepherdson, L (1997) The Mission Critical Browser. http://www.simware.com/whitepapers/salvo/ Accessed 30/07/97

Stobart, S. (1995) Introduction to the Internet, Sunderland University Publication. Vine, D (1997) ISPY : Gathering Business Intelligence. Internet World March Visali S (1997) Information a la carte Information Age October pp 14-16 Walsham, G. (1993) Interpreting Information Systems in Organisations, Chichester: Wiley Ward, A. (1996) Intranet, Information Age. December 1996, pp 42 -53. 